was US\$ 5.4-6.3 billion per year. For the countries in the Arabian Peninsula including Jordan, Kuwait, Qatar, Saudi Arabia, Egypt, and Lebanon, the prevalence rates range from 4.6\% to $23.6 \%$ for CDI isolates. Saudi Arabia has the lowest rate $(4.6 \%)$ among these countries. A remarkable increase in nosocomial CDI cases in the adult ICU at King Abdulaziz Medical City was observed between 18 January and 11 March 2018.The aim of our study was to decrease the incidence of CDI in adult ICUs by implementing evidence-based interventions.

Methods Cluster investigations were done; a time, place, and person table created, brain storming to identify the possible risk factors was evoked by the investigative team, a root cause analysis/fishbone diagram was pulled, and corrective actions were formulated.

Clinicians: hand hygiene (soap and water), use of gloves, barrier precautions, prompt identification and early treatment of CDI cases were applied.

Environmental: proper cleaning, housekeeping protocol review, and types of disinfectants used were highlighted.

Surveillance: outcome surveillance of CDI cases was added to the patient safety annual report plan.

Administration: antibiotic stewardship program (ASP), managing staff shortage, and reinforcement of the guidelines were essential.

Results In the first quarter, the rate of CDI was 2.1 per 1000 patient-days, which dropped to 0.9, 0.4, and 0.4 per 1000 patient-days, respectively, in the second, third, and fourth quarters of 2018.

Conclusion There was a significant reduction in the number of CDI cases after timely and appropriate actions were taken. However, maintaining a zero rate was challenging. Continuous monitoring, ongoing data collection, and education were considered key to reducing CDI.

\section{IMPLEMENTATION OF AN ONCOLOGY ELECTRONIC REFERRAL SYSTEM TO IMPROVE ACCESS TO CARE FOR CHILDREN WITH CANCER (IMPACT): A QUALITY IMPROVEMENT INITIATIVE FROM A SINGLE CENTER}

Wasel Jastaniah, Taghreed Justinia, Saleh Maneea Allogmani, Riyadh Maneea Alloqmani, Basim Alsaywid, Amal Talal Alnakhli. College of Medicine, Umm Al Qura University, King Saud bin Abdulaziz University for Health Sciences, Ibin Senna

\subsection{6/bmjoq-2019-PSF.29}

Background Delayed access to cancer care has been associated with early childhood cancer death. Improving timely access to cancer care is the first important step in the cancer treatment journey. We implemented an electronic referral system (e-RS) at the Princess Noorah Oncology Center (PNOC) to improve timely access to cancer care. This study aimed to assess the impact of implementing an e-RS on timely access to cancer care.

Methods This was an observational retrospective analytical cross-sectional study of 399 pediatric oncology patients selected through a consecutive non-probability sampling technique to review the turnaround time (TAT) of children with cancer diagnosed 12 months before (manual referral system) and 12 months after implementation of the e-RS.

Results Of the 399 pediatric oncology patients diagnosed between January 2014 and December 2015, 59.91\% were male and $40.09 \%$ were female, with a median age for both sexes of 5.0 years (IQR 2.5-9.0 years). 96.3\% of the patients were Saudi and $3.7 \%$ were non-Saudi. The median processing time of the manual referral system was 1075 minutes (IQR 145-1498) while the median processing time for e-RS was 125 minutes (IQR 53-1013). By applying a Mann-Whitney test of significance, the TAT between the two systems was significant $(\mathrm{p}=0.0001)$.

Conclusion Implementing an e-RS improved referral TAT. As a result, pediatric oncology patients had improved timely access to cancer care at PNOC based on TAT standards. The impact of this improvement on outcomes will be reported separately.

\section{PREVALENCE OF HEPATITIS C VIRUS INFECTION AMONG A POPULATION TESTED AT KING ABDULAZIZ MEDICAL CITY, JEDDAH, IN 2018}

Mohammad Abdel Aziz, Fayssal Farahat, Majid AlThaqafy, John Ossenkopp, Lamiaa AlGhamdi, Asim AlSaedi. Infection Prevention and Control King Abdulaziz Medical City, Jeddah

\subsection{6/bmjoq-2019-PSF.30}

Background Hepatitis $\mathrm{C}$ virus (HCV) is one of the three major bloodborne infections. HCV infection has a devastating outcome, yet is a curable disease. The World Health Organization (WHO) targets elimination of the disease by 2030. This target cannot be achieved without screening and case detection. This retrospective study aimed to investigate the prevalence of HCV infection among people tested at King Abdulaziz Medical City, Jeddah (KAMC-J), during 2018.

Methods Results of the HCV antibody enzyme-linked immunosorbent assay (ELISA) tests conducted during 2018 at KAMC-J were obtained from the Electronic Medical System (BestCare). The HCV RNA test results were reviewed through the HCV surveillance database available in the Infection Prevention and Control Department. Data were also collected on demographic variables (age, sex, and nationality). Statistical analysis was done with IBM SPSS version 24.

Results A total of $5425 \mathrm{HCV}$ antibody tests were performed in 2018. More than half of the samples were for females (54.4\%, $\mathrm{n}=2953)$. The overall prevalence of positive $\mathrm{HCV}$ antibodies among the tested population was $1.5 \% \quad(n=82)$. Reactive HCV antibodies were higher among females $(1.6 \%$, $\mathrm{n}=46)$ than males $(1.5 \%, \mathrm{n}=36)$. The prevalence significantly increased with age from $0.3 \%(n=6)$ among people younger than 25 years up to $6.2 \%(n=42)$ among those older than 70 years. HCV positive antibody prevalence was significantly higher among Saudi $(1.8 \%, \mathrm{n}=79)$ than non-Saudi $(0.3 \%$, $\mathrm{n}=3$ ) populations. Of the 82 cases with positive $\mathrm{HCV}$ antibodies, $49(59.8 \%)$ cases were newly diagnosed, of which $30.6 \%(n=15)$ had reactive HCV RNA. Only two people were $\mathrm{HCV} / \mathrm{HBV}$ co-infected.

Conclusion This study demonstrates the importance of HCV screening of high-risk populations, including military personnel, and the need for early intervention in order to achieve the WHO target of eliminating the disease by 2030 . 